\title{
Screening of Lignocellulose-Degrading Superior Mushroom Strains and Determination of Their CMCase and Laccase Activity
}

\author{
Li Fen, ${ }^{1}$ Zhu Xuwei, ${ }^{1}$ Li Nanyi, ${ }^{2}$ Zhang Puyu, ${ }^{1}$ Zhang Shuang, ${ }^{1}$ Zhao Xue, \\ Li Pengju, ${ }^{1}$ Zhu Qichao, ${ }^{3}$ and Lin Haiping ${ }^{1}$ \\ ${ }^{1}$ The Nurturing Station for the State Key Laboratory of Subtropical Silviculture, Provincial Engineering Laboratory of \\ Biopesticide Preparation, Zhejiang A\&F University, Linian 311300, China \\ ${ }^{2}$ School of Agricultural and Food Science, Zhejiang A\&F University, Linian 311300, China \\ ${ }^{3}$ Qingyuan Forestry Bureau of Zhejiang, Qingyuan 323800, China \\ Correspondence should be addressed to Lin Haiping; zjlxylhp@163.com
}

Received 10 November 2013; Accepted 24 December 2013; Published 12 February 2014

Academic Editors: C. Cravo-Laureau and K. Ohmiya

Copyright (C) 2014 Li Fen et al. This is an open access article distributed under the Creative Commons Attribution License, which permits unrestricted use, distribution, and reproduction in any medium, provided the original work is properly cited.

\begin{abstract}
In order to screen lignocellulose-degrading superior mushroom strains ten strains of mushrooms (Lentinus edodes939, Pholiota nameko, Lentinus edodes868, Coprinus comatus, Macrolepiota procera, Auricularia auricula, Hericium erinaceus, Grifola frondosa, Pleurotus nebrodensis, and Shiraia bambusicola) were inoculated onto carboxymethylcellulose agar-Congo red plates to evaluate their ability to produce carbomethyl cellulase (CMCase). The results showed that the ratio of transparent circle to mycelium circle of Hericium erinaceus was $8.16(P<0.01)$ higher than other strains. The filter paper culture screening test showed that Hericium erinaceus and Macrolepiota procera grew well and showed extreme decomposition of the filter paper. When cultivated in guaiacol culture medium to detect their abilities to secrete laccase, Hericium erinaceus showed the highest ability with the largest reddish brown circles of $4.330 \mathrm{~cm}$. CMCase activity determination indicated that Coprinus comatus and Hericium erinaceus had the ability to produce CMCase with $33.92 \mathrm{U} / \mathrm{L}$ on the 9th day and $22.58 \mathrm{U} / \mathrm{L}$ on the 10th day, respectively, while Coprinus comatus and Pleurotus nebrodensis had the ability to produce laccase with $496.67 \mathrm{U} / \mathrm{L}$ and $489.17 \mathrm{U} / \mathrm{L}$ on the 16th day and 18th day. Based on the results, Coprinus comatus might be the most promising lignocellulose-degrading strain to produce both CMCase and laccase at high levels.
\end{abstract}

\section{Introduction}

Lignocellulose is an abundant and renewable source of carbohydrates that can be converted into value-added products in the earth. It is estimated that $6.0 \times 10^{9}$ tons of lignocellulose are generated by photosynthesis each year all over the world, but only about $20 \%$ are used for conversion into energy and food [1]. Many lignocellulose litters such as straws and sawdusts are produced each year in industry and agriculture. Therefore it is of great importance to make these wastes available to reduce environmental pollution and stabilize the development of bioenergy [2].

Agricultural residues are rich in lignocellulosic compounds whose disposal and handling are problematic due to their complex structure and decomposition properties [3, 4]. Lignin wraps around cellulose and hemicelluloses fibers, which inhibits the degradation of both, so it is necessary to remove the lignin to facilitate the use of cellulose and hemicelluloses as a source for bioenergy. Recently, chemical and physical methods of pretreating lignocellulosic compounds have been used to expose the underlying cellulose and hemicelluloses such as radicalization, steam explosion, puffing, acid, and alkali [5]. Unfortunately, these methods consume high amounts of energy and cause pollutants [6]. So it is pressing to find a high-efficiency, energy-saving, and environment-friendly way to break down cellulose and hemicelluloses.

Some microorganisms, especially some edible and medicinal mushrooms, produce a complete set of enzymes capable of efficient degradation of native cellulose and lignin, so screening of lignocellulose-degrading superior mushroom strains has become a significant project in the process of reusing lignocellulose wastes. Enzymes which are responsible 
TABLE 1: Ratio of transparent circle to mycelium circle of 10 strains under CMC culture.

\begin{tabular}{lccc}
\hline Strains & Transparent circle diameter $(d 1) / \mathrm{cm}$ & Mycelium circle diameter $(d 2) / \mathrm{cm}$ & $d 1 / d 2$ \\
\hline Lentinus edodes939 & $3.87 \pm 0.22$ & $3.49 \pm 0.09$ & $1.11 \pm 0.06^{\mathrm{C}}$ \\
Pholiota nameko & $5.18 \pm 0.11$ & $5.04 \pm 0.27$ & $1.03 \pm 0.06^{\mathrm{C}}$ \\
Lentinus edodes868 & $4.78 \pm 0.22$ & $3.84 \pm 0.22$ & $1.24 \pm 0.06^{\mathrm{C}}$ \\
Coprinus comatus & $3.34 \pm 0.08$ & $2.42 \pm 0.22$ & $1.89 \pm 0.17^{\mathrm{C}}$ \\
Macrolepiota procera & $4.32 \pm 0.21$ & $2.32 \pm 0.30$ & $2.30 \pm 0.30^{\mathrm{B}}$ \\
Auricularia auricula & $1.41 \pm 0.10$ & $0.62 \pm 0.17$ & $8.16 \pm 0.56^{\mathrm{A}}$ \\
Hericium erinaceus & $4.95 \pm 0.49$ & $0.61 \pm 0.09$ & $2.26 \pm 0.05^{\mathrm{B}}$ \\
Grifola frondosa & $3.84 \pm 0.19$ & $1.70 \pm 0.11$ & $0.00 \pm 0.00^{\mathrm{D}}$ \\
Pleurotus nebrodensis & $0.00 \pm 0.00$ & $2.76 \pm 0.51$ & $0.00 \pm 0.00^{\mathrm{D}}$ \\
Shiraia bambusicola & $0.00 \pm 0.00$ & $5.91 \pm 0.72$ & \\
\hline
\end{tabular}

Different capital letters followed by the data in the table mean the significant difference at 0.01 level.

The data represent the average of three independent experiments.

for cellulose degradation are hydrolytic [7]. The cellulosehydrolysing enzymes (i.e., cellulases) are divided into three major groups: endoglucanases, cellobiohydrolases (exoglucanases), and $\beta$-glucosidases $[8,9]$. Lignin degradation mainly depends on oxidative enzymes [10], including lignin peroxidase, manganese-dependent peroxidase, and laccase which are well studied. In wood-destroying white rot fungi, laccases are the most important components of the lignolytic complex responsible for decomposition of lignin, which is one of the most-difficult-to-be-decomposed polymers in nature $[11,12]$.

Many researches focus on the fields of screening of lignocellulose-degrading superior strains, while many others focus on high production mushrooms all over the world, but very few put the two areas together. At present, microbes used in cellulose degradation are mainly fungi, of which Trichoderma, Aspergillus, Rhizopus, and Myrothecium have been studied the most [3]. Since edible and medicinal fungi can convert lignincellulose to form fruiting bodies, it indicated they can decompose lignocellulose to micromolecular nutrient substance for fungi to be utilized. Thus studies on screening of lignocellulose-degrading superior edible fungi play an important role [13]. In this study 10 strains of mushrooms were investigated for their ability to degrade lignocellulose. The purpose and significance of the research are obtaining some strains of mushrooms that have high ability to degrade lignocellulose. So in the process of cultivating them on lignocellulose litters, mushroom production and raw material to produce bioenergy could be obtained at the same time.

\section{Materials and Methods}

2.1. Fungal Strains and Culture Media. Ten commercial strains (Lentinus edodes939 ACCC51655, Pholiota nameko ACCC51896, Lentinus edodes868 ACCC51717, Coprinus comatus ACCC51773, Macrolepiota procera ACCC51531, Auricularia auricula ACCC51604, Hericium erinaceus ACCC51322, Grifola frondosa ACCC51962, Pleurotus nebrodensis ACCC51498, and Shiraia bambusicola ACCC50027 (Agricultural Culture Collection of China)) were subjected to screening experiments.
All strains were seeded on potato dextrose agar medium (PDA: potato $200 \mathrm{mg} / \mathrm{mL}$ glucose $20 \mathrm{mg} / \mathrm{mL}$ and agar $20 \mathrm{mg} /$ $\mathrm{mL}$ ) slants and discs and incubated at $26^{\circ} \mathrm{C}$ for $7-10$ days until colonies appeared which were prepared for the following experiments.

Carboxymethylcellulose (CMC)-agar culture medium contained $10 \mathrm{mg} / \mathrm{mL}$ sodium CMC, $4 \mathrm{mg} / \mathrm{mL}\left(\mathrm{NH}_{4}\right)_{2} \mathrm{SO}_{4}$, $2 \mathrm{mg} / \mathrm{mL} \mathrm{KH}_{2} \mathrm{PO}_{4}$ and $20 \mathrm{mg} / \mathrm{mL}$ agar [14], in which sodium carboxymethylcellulose $\left(\mathrm{CMC}_{\mathrm{Na}}\right)$ was the sole carbon source for the fungus.

Filter paper culture medium contained $1 \mathrm{mg} / \mathrm{mL}$ $\left(\mathrm{NH}_{4}\right)_{2} \mathrm{SO}_{4}, 1 \mathrm{mg} / \mathrm{mL} \mathrm{KH}_{2} \mathrm{PO}_{4}, 0.7 \mathrm{mg} / \mathrm{mL} \mathrm{MgSO}_{4} \cdot 7 \mathrm{H}_{2} \mathrm{O}$, $0.5 \mathrm{mg} / \mathrm{mL} \mathrm{NaCl}$, and $6 \mathrm{~cm} \times 1 \mathrm{~cm}$ filter paper [14, 15].

CMCase production medium contained $10 \mathrm{mg} / \mathrm{mL}$ $\mathrm{CMC}_{\mathrm{Na}}, 4 \mathrm{mg} / \mathrm{mL}\left(\mathrm{NH}_{4}\right)_{2} \mathrm{SO}_{4}, 2 \mathrm{mg} / \mathrm{mL} \mathrm{KH} \mathrm{PO}_{4}, 0.5 \mathrm{mg} /$ $\mathrm{mL} \mathrm{MgSO}_{4} \cdot 7 \mathrm{H}_{2} \mathrm{O}, 10 \mathrm{mg} / \mathrm{mL}$ peptone, and $5 \mathrm{mg} / \mathrm{mL}$ beef extract [3].

Guaiacol color culture medium contained $1.0 \mathrm{mg} / \mathrm{mL}$ guaiacol, $0.1 \mathrm{mg} / \mathrm{mL} \quad \mathrm{C}_{4} \mathrm{H}_{12} \mathrm{~N}_{2} \mathrm{O}_{6}, 2.6 \mathrm{mg} / \mathrm{mL}$ peptone, $0.5 \mathrm{mg} / \mathrm{mL} \quad \mathrm{MgSO}_{4} \cdot 7 \mathrm{H}_{2} \mathrm{O}, 1 \mathrm{mg} / \mathrm{mL} \quad \mathrm{KH}_{2} \mathrm{PO}_{4}, 0.2 \mathrm{mg} / \mathrm{mL}$ $\mathrm{Na}_{2} \mathrm{HPO}_{4}$, and $20 \mathrm{mg} / \mathrm{mL}$ agar [16].

Laccase production medium was equal to PDA without agar.

All media were autoclaved at $121^{\circ} \mathrm{C}$ for 20 minutes.

2.2. CMC Culture Screening. Five $\mathrm{mm}$ disc diameter was taken from the PDA plates as inoculated on CMC plates and then grew at $26^{\circ} \mathrm{C}$ for about one week until mycelium was proper to measure. Afterwards, the plates were dyed by adding $20 \mathrm{~mL}$ Congo red $(1 \mathrm{mg} / \mathrm{mL})$, and three hours later, $1 \mathrm{~mol} / \mathrm{L} \mathrm{NaCl}$ was added to decolorize them. The diameters of transparent circle and mycelium circle on each plate were measured.

The ratio of transparent circle to colony diameter was then calculated to estimate the ability of the fungi to degrade cellulose [17]. The experiment was repeated three times. The data presented in Table 1 represent mean values \pm standard deviation.

2.3. Filter Paper Culture Screening. Each $15 \times 150 \mathrm{~mm}$ test tube was filled with $5 \mathrm{~mL}$ filter paper culture medium so that there 


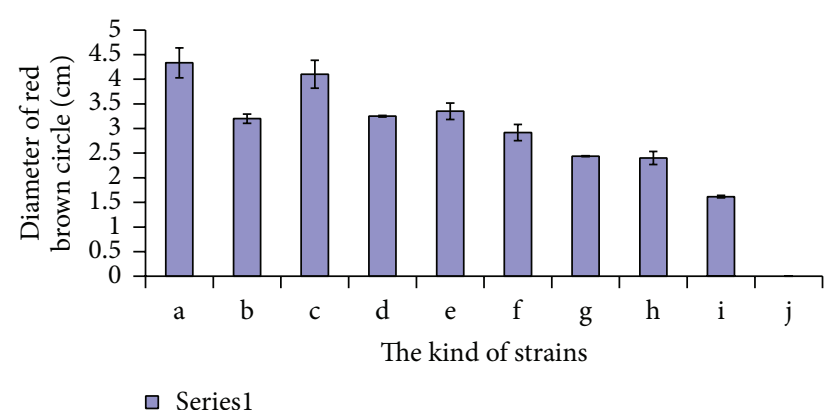

FIGURE 1: Diameter of red brown circle of 10 strains. One week after inoculating on guaiacol color culture media. a: Hericium erinaceus, b: Coprinus comatus, c: Lentinus edodes868, d: Lentinus edodes939, e: Pleurotus nebrodensis, f: Auricularia auricular, g: Pholiota nameko, h: Macrolepiota procera, i: Shiraia bambusicola, and j: Grifola frondosa. The data represent the average of three independent experiments.

for sure was $1 \mathrm{~cm}$ of $6 \times 1 \mathrm{~cm}$ filter paper out of the liquid. Strains which were inoculated into the filter paper culture medium were kept at the surface of the liquid and in the filter paper and then cultivated statically at $26^{\circ} \mathrm{C}$ for about half a month. The experiment was repeated three times.

2.4. Guaiacol Color Culture Screening. One mycelium agar disc (five $\mathrm{mm}$ diameter), cut from the growing edge of each strain PDA culture, was used to inoculate each plate and incubated at $26^{\circ} \mathrm{C}$ for about one week and then monitored for the appearance of a reddish brown circle. The diameter of the reddish brown circle was measured to identify laccase secreting ability of the stains. The experiment was repeated three times. The data presented in Figure 1 represent values \pm standard deviation.

2.5. Fermentation. Superior cellulose degradation strains were selected based on plate culture results and inoculated into $250 \mathrm{~mL}$ Erlenmeyer flasks containing $50 \mathrm{~mL}$ of CMCase production medium. Five mycelium agar discs (five $\mathrm{mm}$ diameter) cut from the growing edge of each strain 7-day PDA culture were used to inoculate each flask. The strains were cultivated in a rotary shaker at $120 \mathrm{rev} \cdot \mathrm{min}^{-1}$ at $26^{\circ} \mathrm{C}$ and were sampled each day for five days. All experiments were performed with three replicates.

2.6. Enzyme Assay. Carboxymethylcellulase (CMCase) was estimated by using $1 \%$ carboxymethyl cellulose as the sole carbon source. The reaction mixture was composed of $0.2 \mathrm{~mL}$ enzyme sample and $1.8 \mathrm{~mL} \mathrm{CMC} \mathrm{(1 \% )} \mathrm{dissolved} \mathrm{in} 0.1 \mathrm{~mol} / \mathrm{L}$ sodium acetate buffer ( $\mathrm{pH}$ 4.8). The reaction was kept at $50^{\circ} \mathrm{C}$ for 30 minutes. The amount of reducing sugar was determined by the DNS(3,5-dinitrosalicylic) method [18], using glucose as a standard at $550 \mathrm{~nm}$. As a control, $1.8 \mathrm{~mL}$ of sodium acetate buffer was used instead of CMC following the same treatment [19]. Enzymatic activity, expressed as $\mathrm{U} / \mathrm{mL}$, was defined as the amount of enzyme producing $1 \mu \mathrm{g}$ of product per minute per $\mathrm{mL}$ of substrate extracted. Each

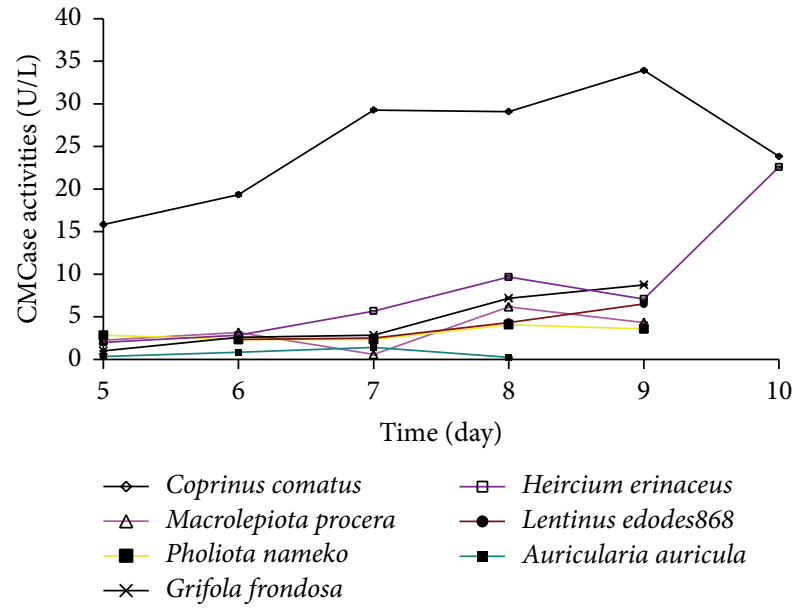

FIgURE 2: Time courses of CMCase activity of 7 strains. Five mycelium agar discs ( $5 \mathrm{~mm}$ diameter) were used as the inoculum. Cultivated in CMC liquid medium, $1 \mathrm{~mL}$ Zymotic fluid of the strains was taken out of the flask for experiment each day. The data represent the average of three independent experiments.

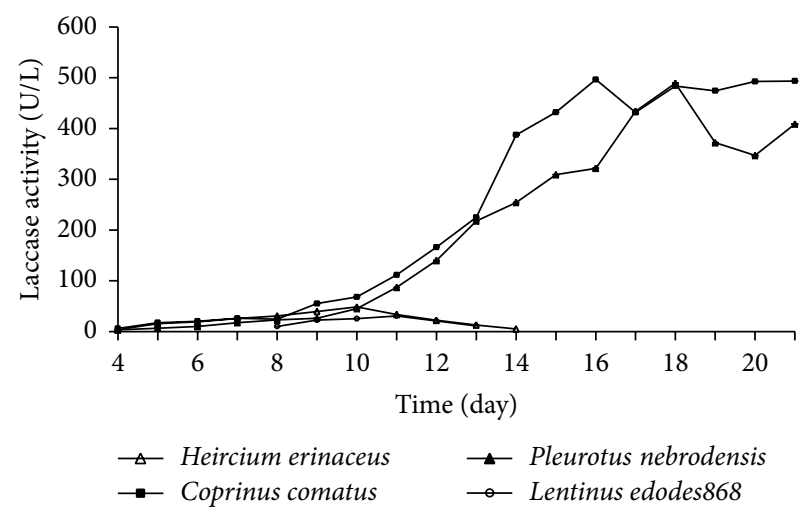

FIGURE 3: Time courses of laccase activity of 4 strains. Five mycelium agar discs ( $5 \mathrm{~mm}$ diameter) were used as the inoculum. Cultivated in potato dextrose liquid medium, $1 \mathrm{~mL}$ Zymotic fluid of the strains was taken out of the flask for experiment each day. The data represent the average of three independent experiments.

same experiment was repeated three times. The data in Figure 2 represent the average of three independent experiments.

The strains which showed the capacity to secrete laccase in guaiacol color culture screening were used to determine laccase activity and were conducted in $3 \mathrm{~mL}$ reaction mixtures consisting of $2.7 \mathrm{~mL}$ of $0.1 \mathrm{~mol} / \mathrm{L}$ sodium acetate buffer ( $\mathrm{pH} 4.5$ ), $0.2 \mathrm{~mL}$ of $1 \mathrm{mmol} / \mathrm{L} \mathrm{2,20'}$-azinobis(3ethylbenzothiazoline-6-sulfonic acid) (ABTS) solution, and $0.1 \mathrm{~mL}$ extracellular culture supernatant. The reaction was monitored by measuring the change in $420 \mathrm{~nm}$ for $3 \mathrm{~min}$. One unit of enzyme activity is defined as the amount of oxidation of ABTS followed by 0.1 increase in absorbance at $420 \mathrm{~nm}$. The molar absorption coefficient of oxidized ABTS is $3.6 \times$ $10^{4} \mathrm{~L} /(\mathrm{mol} \cdot \mathrm{cm})[1]$. Each same experiment was repeated three times. The data in Figure 3 represent the average of three independent experiments. 


\section{Results}

3.1. CMC Culture and Filter Paper Culture Screening. Mushrooms were cultivated in CMC-agar culture to determine their ability to use $\mathrm{CMC}_{\mathrm{Na}}$ as the sole carbon source. All strains tested showed different mycelial growth in CMC culture. Based on estimations of their mycelium diameters, Shiraia bambusicola grew best with mycelium growth diameter of $5.91 \mathrm{~cm}$, followed by Pholiota nameko, Lentinus edodes868, and Lentinus edodes939, where mycelium growth diameters were $5.04 \mathrm{~cm}, 3.84 \mathrm{~cm}$, and $3.49 \mathrm{~cm}$, respectively. Hericium erinaceus had the smallest mycelium growth diameter of $0.61 \mathrm{~cm}$ followed by Auricularia auricular with $0.62 \mathrm{~cm}$ mycelium diameter. However, a rather different case was observed in the transparent circles that represent cellulose degradation. Shiraia bambusicola and Pleurotus nebrodensis showed no transparent circle, and Auricularia auricula with $1.41 \mathrm{~cm}$ transparent circle became the third inferior candidate, while Pholiota nameko followed by Hericium erinaceus had the largest transparent circles $(5.18 \mathrm{~cm}$ and $4.95 \mathrm{~cm}$, resp.). To sum up, Hericium erinaceus had the highest ratio of transparent circle diameter to mycelium circle diameter $(d 1 / d 2)$ with the datum of 8.16 . Big differences were observed among $d 1 / d 2$ of 10 different strains (Table 1 ).

The filter paper culture screening test showed that all strains grew slowly with evidence of mycelium extension in approximately half a month. The growth results also varied from strain to strain. Hericium erinaceus and Macrolepiota procera grew well in the filter paper culture medium and showed extreme decomposition of the filter paper, while Grifola frondosa, Shiraia bambusicola, and Coprinus comatus showed scarce biomass growth and without observable degradation. Some strains such as Lentinus edodes 868 and Macrolepiota procera showed less than optimal growth but were still able to decompose the filter paper. All strains were divided into four groups according to the degree of decomposing the filter paper. Hericium erinaceus and Macrolepiota procera decomposed the filter paper in a good manner. Most of the strains decomposed the filter paper ordinarily, including Lentinus edodes868, Pleurotus nebrodensis, Lentinus edodes939, Auricularia auricula, and Coprinus comatus. While Grifola frondosa and Pholiota nameko faintly decomposed the filter paper, Shiraia bambusicola showed no degradation ofthe filter paper (Table 2). As shown in Tables 1 and 2, the results of CMC culture and filter paper culture screening experiment are approximately consistent.

3.2. Guaiacol Color Culture Screening. Guaiacol is one of the substrates of laccase and the degradation product of guaiacol is rufous. Therefore we used the red brown color to indicate the laccase production by the strains and the diameter of the colored circle to estimate the quantity of laccase produced. All plates showed a reddish brown color after one week except for the plate with Grifola frondosa which was reddish brown the second day after inoculation. As time went by, the reddish brown circle expanded and eventually stabilized. Hericium erinaceus, Lentinus edodes868, Pleurotus nebrodensis, and Coprinus comatus had large reddish brown circles. Their reddish brown circle diameters were $4.33 \mathrm{~cm}$,
TABLE 2: The decomposing degree of 10 strains on filter paper.

\begin{tabular}{lc}
\hline Strains & The decomposing degree \\
\hline Lentinus edodes 939 & $++^{\mathrm{b}}$ \\
Pholiota nameko & $+^{\mathrm{c}}$ \\
Lentinus edodes868 & ++ \\
Coprinus comatus & ++ \\
Macrolepiota procera & +++ \\
Auricularia auricula & ++ \\
Hericium erinaceus & $+++^{\mathrm{a}}$ \\
Grifola frondosa & + \\
Pleurotus nebrodensis & ++ \\
Shiraia bambusicola & $0^{\mathrm{d}}$ \\
\hline
\end{tabular}

$a_{+}++$stands for the strain decomposed the filter paper in a good manner.

$\mathrm{b}_{++}$stands for the strain decomposed the filter paper in a common manner.

${ }^{c}+$ stands for the strain faintly decomposed the filter paper.

${ }^{\mathrm{d}} 0$ stands for the strain decomposed no filter paper.

The data represent the average of three independent experiments.

$4.10 \mathrm{~cm}, 3.35 \mathrm{~cm}$, and $3.20 \mathrm{~cm}$, respectively (Figure 1). So these four strains were selected to determine the activity of laccase.

3.3. Determination of CMCase Activity. Based on the quantitative analysis of transparent circles to mycelial growth diameter, we were more interested in the numerical value of enzyme activity. The determination showed that CMCase activity of Coprinus comatus was obviously higher than others. As the incubation time increased, CMCase activity also increased and the peak (33.92 U/L) appeared on day 9. Hericium erinaceus showed the second highest CMCase activity. The variation rule of CMCase activity of Hericium erinaceus seemed similar to Coprinus comatus. However, the activity produced two peaks: one on day eight and the other on day 10. Furthermore, the CMCase activity increased dramatically on day 10 by nearly threefold more than that on day nine and 2.34-fold of the first peak. Pholiota nameko showed no significant CMCase activity increase during the cultivation. Although CMCase activity of Grifola frondosa and Macrolepiota procera gradually increased, the level of activity remained low. It seemed that Auricularia auricular was deficient in CMCase production (Figure 2). CMCase activities of other strains which were not shown in Figure 2 were not detected. The CMCase activity is also approximately consistent with of the results of CMC culture and filter paper culture screening experiment.

3.4. Determination of Laccase Activity. The laccase activities of superior strains that had been selected based on Guaiacol color culture screening are shown chronologically in Figure 3. Coprinus comatus and Pleurotus nebrodensis displayed much higher laccase activities than Hericium erinaceus and Lentinus edodes868. Laccase activities of Coprinus comatus and Pleurotus nebrodensis reached their peaks with $496.67 \mathrm{U} / \mathrm{L}$ and 489.17 U/L at day 16 and day 18, respectively, while Hericium erinaceus reached its peak of $48.54 \mathrm{U} / \mathrm{L}$ at day 10 and Lentinus edodes 868 reached its peak of $30.88 \mathrm{U} / \mathrm{L}$ at day 11. 
Coprinus comatus has not only the highest CMCase activity but also the highest laccase activity. It is a kind of delicious mushroom at the same time, so Coprinus comatus is a promising mushroom strain to be used in the development of bioenergy.

\section{Discussion}

Cellulose, a homopolysaccharide composed of $\beta$-D-glucopyranose units, linked by $\beta$ - $(1 \rightarrow 4)$-glycosidic bonds can be converted to cellobiose and glucose by cellulase [4]. Congo red can bind to polysaccharides like cellulose but cannot bind to saccharides to form red compounds. Therefore when cellulose was hydrolyzed to monosaccharide, the unconjugated Congo red was eluted by saline solution and then the transparent circles were apparent in culture plates [20]. If cellulose was not hydrolyzed, it could not bound to Congo red and therefore could not be eluted and remained red. Hankin and Anagnostakis [21] found that transparent circles were produced around the colonies in some cellulose culture medium, while Teather and Wood [22] hold that there, to some extent, existed a linear relationship between the transparent circle size and cellulase activity. However, some strains that were tested in this investigation such as Lentinus edodes939, Lentinus edodes868, and Pholiota nameko showed bigger transparent circles in CMC culture screening but indicated no or low activity in the following CMCase activity test. On the other hand, Coprinus comatus with inferior results in CMC culture screening showed the highest CMCase activity in carbomethyl cellulase activity (CMCase) determination. About $d 1 / d 2$, the situation was similar. For example, Coprinus comatus had the highest CMCase activity and was obviously higher than others, but the $d 1 / d 2$ of Coprinus comatus was inferior. Another case is Hericium erinaceus; it had the highest $d 1 / d 2$, while CMCase activity was second, but the latter increased remarkably after $9 \mathrm{~d}$ during fermentation. In addition, the results of CMC culture and filter paper culture screening experiment are approximately consistent. Therefore, the transparent circle, $d 1 / d 2$, degradative degree of filter paper, or CMCase activity alone cannot determine the fungi's ability to secrete cellulase. It is better to combine the results of four aspects to choose the cellulose degrading superior mushroom strains.

Although it indicated that Coprinus comatus and Hericium erinaceus were preferential candidates of degrading cellulose, it is only under our technique of fermentation. So it is necessary to optimize the liquid culture technique in order to increase the activity of cellulose next stage. And at the same time, the cellulase activity of the strains when they grow on the solid lignocellulose waste is worthy of being studied, because the microorganisms grow under such conditions closer to their natural habitat and can possibly produce larger amounts or higher activity of extracellular enzymes.

In fungi, laccase is known as a ligninolytic enzyme and appears to be involved in fruiting body formation, fungal plant-pathogen/host interaction, and stress defense. Since laccase plays a major role in fungal degradation of lignolytic compounds, its activity to some extent reflects the ability of the fungus to decompose lignocellulosic materials. In addition to a remarkably low substrate specificity, laccase has other properties that make this enzyme potentially useful for biotechnological application, like laccase does not need the addition or synthesis of low molecule weight cofactor. Although laccases have been found in many species, the information from the edible fungi is less reported [23].

In this report, we found that Coprinus comatus and Hericium erinaceus had good ability of producing CMCase, while Coprinus comatus and Pleurotus nebrodensis had good ability of producing laccase. So Coprinus comatus was able to produce both enzymes at high levels. In recent years, It is found that Hericium erinaceus is capable of biodegrading straw [24]. These results may provide a useful tool for screening of high laccase-producing and CMCase-producing strains and offer a fundamental method to study the laccase production capacity of fungi.

As the world energy crisis is becoming increasingly severe, it is necessary and urgent to find a new energy to substitute or complement petroleum energy. Undoubtedly lignocellulose is the optimal candidate. Since microbes play a crucial rule in the process of conversion of lignocellulose to bioenergy, this report may offer a promising mushroom strain to use in the development of bioenergy.

\section{Conflict of Interests}

The authors declare that there is no conflict of interests regarding the publication of this paper.

\section{Acknowledgments}

This work was financially supported by Natural Science Funding Proposal of Zhejiang Province (LY12C14005).

\section{References}

[1] S. J. Sun, J. Z. Liu, K. H. Hu, and H. X. Zhu, "The level of secreted laccase activity in the edible fungi and their growing cycles are closely related," Current Microbiology, vol. 62, no. 3, pp. 871-875, 2011.

[2] S. A. Furlan, L. J. Virmond, D. A. Miers, M. Bonatti, R. M. M. Gern, and R. Jonas, "Mushroom strains able to grow at high temperatures and low $\mathrm{pH}$ values," World Journal of Microbiology and Biotechnology, vol. 13, no. 6, pp. 689-692, 1997.

[3] L. R. Han, S. X. Zhang, C. S. Zhu, and X. Zhang, "Screening and identification of superior fungus degraded cellulose," Journal of Northwest A \& F University, vol. 36, pp. 169-174, 2008 (Chinese).

[4] O. S. Isikhuemhen, N. A. Mikiashvili, and V. Kelkar, "Application of solid waste from anaerobic digestion of poultry litter in Agrocybe aegerita cultivation: mushroom production, lignocellulolytic enzymes activity and substrate utilization," Biodegradation, vol. 20, no. 3, pp. 351-361, 2009.

[5] H. P. Do, R. Q. Song, and Y. Q. Wang, "Comparative study on the lignocellulolytic enzymes produced by fungi," Forestry Science \& Technology, vol. 31, pp. 20-24, 2006.

[6] M. S. Chandra, B. Viswanath, and B. R. Reddy, "Cellulolytic enzymes on lignocellulosic substrates in solid state fermentation by Aspergillus niger," Indian Journal of Microbiology, vol. 47, no. 4, pp. 323-328, 2007. 
[7] T. Kajisa, K. Igarashi, and M. Samejima, "The genes encoding glycoside hydrolase family 6 and 7 cellulases from the brownrot fungus Coniophora puteana," Journal of Wood Science, vol. 55, no. 5, pp. 376-380, 2009.

[8] R. Kumar, S. Singh, and O. V. Singh, "Bioconversion of lignocellulosic biomass: biochemical and molecular perspectives," Journal of Industrial Microbiology and Biotechnology, vol. 35, no. 5, pp. 377-391, 2008.

[9] Y. Liang, J. Yesuf, S. Schmitt, K. Bender, and J. Bozzola, "Study of cellulases from a newly isolated thermophilic and cellulolytic Brevibacillus sp. strain JXL," Journal of Industrial Microbiology and Biotechnology, vol. 36, no. 7, pp. 961-970, 2009.

[10] T. K. Kirk and D. Cullen, "Enzymology and molecular genetics of wood degradation by white-rot fungi," in Environmentally Friendly Technologies for the Pulp and Paper Industry, R. A. Young and M. Akhtar, Eds., pp. 273-308, John Wiley and Sons, New York, NY, USA, 1998.

[11] O. V. Morozova, G. P. Shumakovich, M. A. Gorbacheva, S. V. Shleev, and A. I. Yaropolov, “"Blue” laccases," Biochemistry, vol. 72, no. 10, pp. 1136-1150, 2007.

[12] G. Elegir, D. Bussini, S. Antonsson, M. E. Lindström, and L. Zoia, "Laccase-initiated cross-linking of lignocellulose fibres using a ultra-filtered lignin isolated from kraft black liquor," Applied Microbiology and Biotechnology, vol. 77, no. 4, pp. 809817, 2007.

[13] P. Rani, N. Kalyani, and K. Prathiba, "Evaluation of lignocellulosic wastes for production of edible mushrooms," Applied Biochemistry and Biotechnology, vol. 151, no. 2-3, pp. 151-159, 2008.

[14] X. G. Wang, Q. Yang, and H. Yan, "Screening and identification for cellulose degradation of strain," Heilongjiang Animal Science and Veterinary Medicine, vol. 8, pp. 70-71, 2007 (Chinese).

[15] S. Jahangeer, N. Khan, S. Jahangeer et al., "Screening and characterization of fungal cellulases isolated from the native environmental source," Pakistan Journal of Botany, vol. 37, no. 3, pp. 739-748, 2005.

[16] H. Y. Yu, G. M. Zeng, G. H. Huang, T. J. Hu, and Y. N. Chen, "Screening of lignin- degrading fungi and their enzyme production," Chinese Journal of Applied \& Environmental Biology, vol. 10, no. 5, pp. 639-642, 2004 (Chinese).

[17] Y. S. Lin, "Separate and screen fungus which can biodegrade cellulose and assay its enzyme activity," Acta Scientiarum Naturalium Universitatis Sunyatseni, vol. 43, pp. 82-85, 2004.

[18] G. L. Miller, "Use of dinitrosalicylic acid reagent for determination of reducing sugar," Analytical Chemistry, vol. 31, no. 3, pp. 426-428, 1959.

[19] G. K. Han, H. P. Sang, H. K. Seong, G. P. Hyuk, and M. P. Won, "Detection and recovery of hydrolytic enzymes from spent compost of four mushroom species," Folia Microbiologica, vol. 50, no. 2, pp. 103-106, 2005.

[20] C. Gao, J. Y. Meng, and F. Y. Feng, "Separating and screening on the cellulose-decomposing bacteria and study on its enzyme activity," Animal Husbandry and Feed Science, vol. 4, pp. 17-20, 2008 (Chinese).

[21] L. Hankin and S. L. Anagnostakis, "Solid media containing carboxymethylcellulose to detect Cx cellulase activity of micro organisms," Journal of General Microbiology, vol. 98, no. 1, pp. 109-115, 1977.

[22] R. M. Teather and P. J. Wood, "Use of Congo red-polysaccharide interactions in enumeration and characterization of cellulolytic bacteria from the bovine rumen," Applied and Environmental Microbiology, vol. 43, no. 4, pp. 777-780, 1982.
[23] W. S. Zhang, X. L. Luo, L. Zhu, Y. S. Xiong, and P. Zhu, “The research review of laccase in the edible fungus," Edible Fungi of China, vol. 28, pp. 8-10, 2009 (Chinese).

[24] Y. R. Li, G. Y. Zhou, Q. X. Hu, and Z. S. Feng, "Research advance on biodegradation of lignin by mushroom," Edible Fungi of China, vol. 28, pp. 3-6, 2009 (Chinese). 

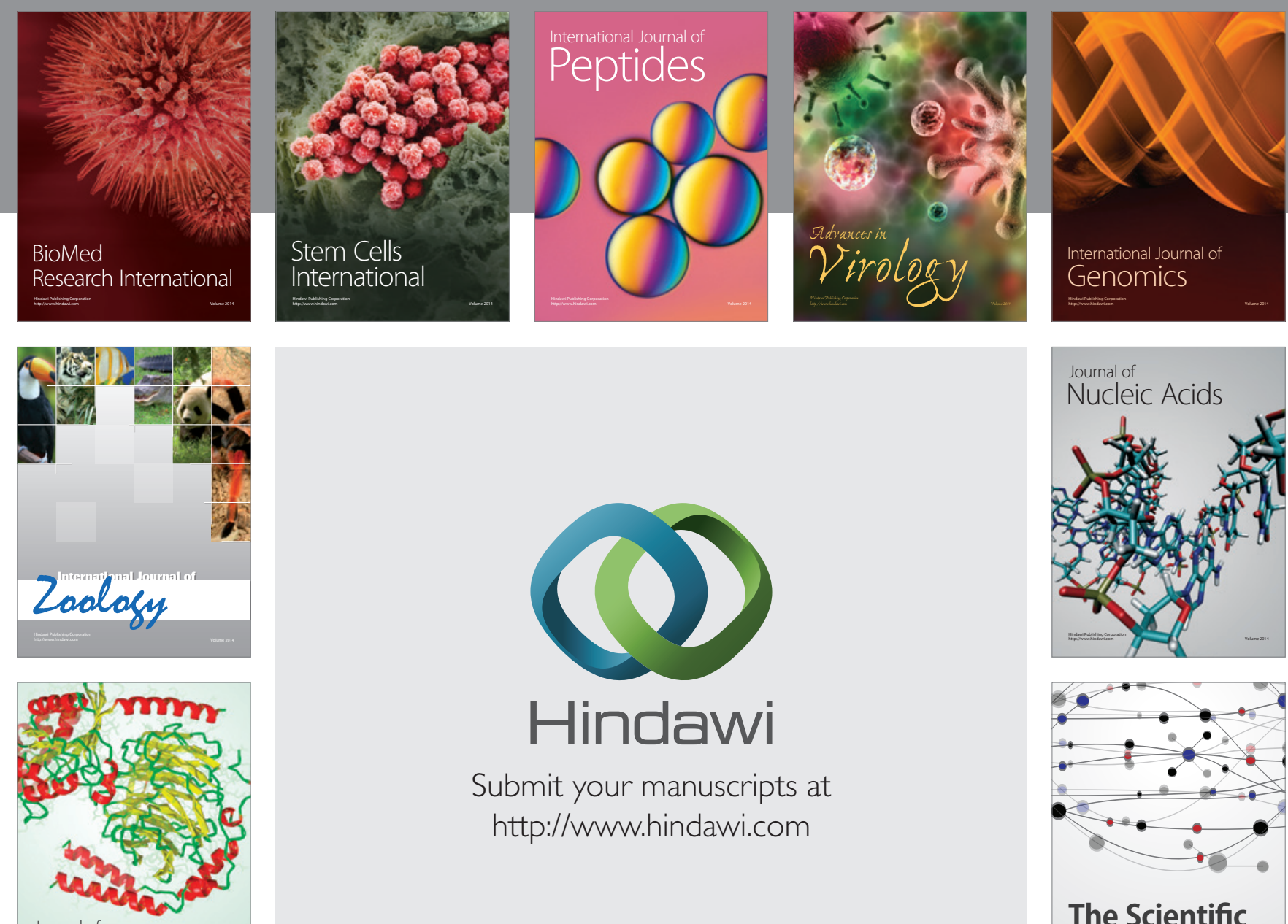

Submit your manuscripts at

http://www.hindawi.com

Journal of
Signal Transduction
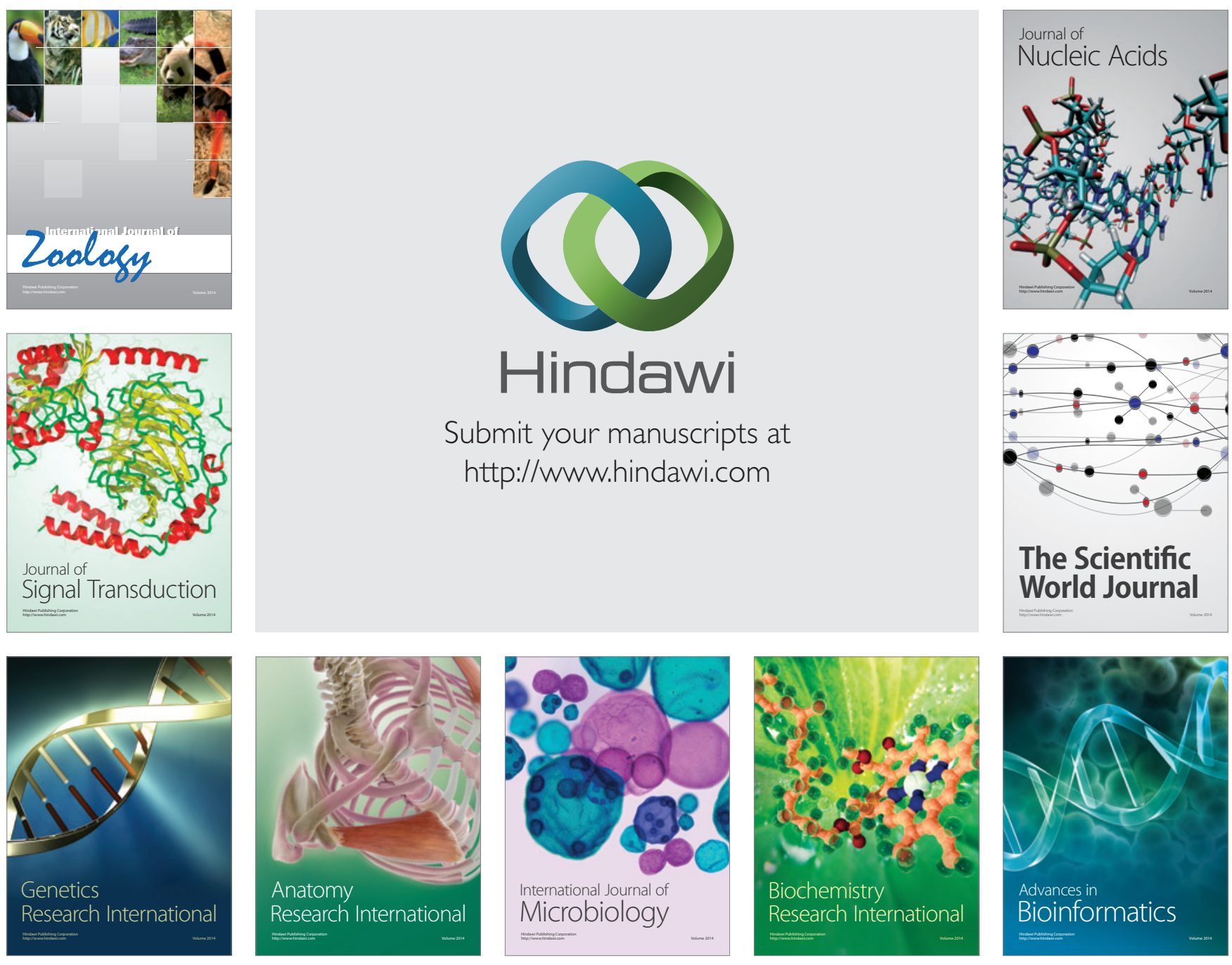

The Scientific World Journal
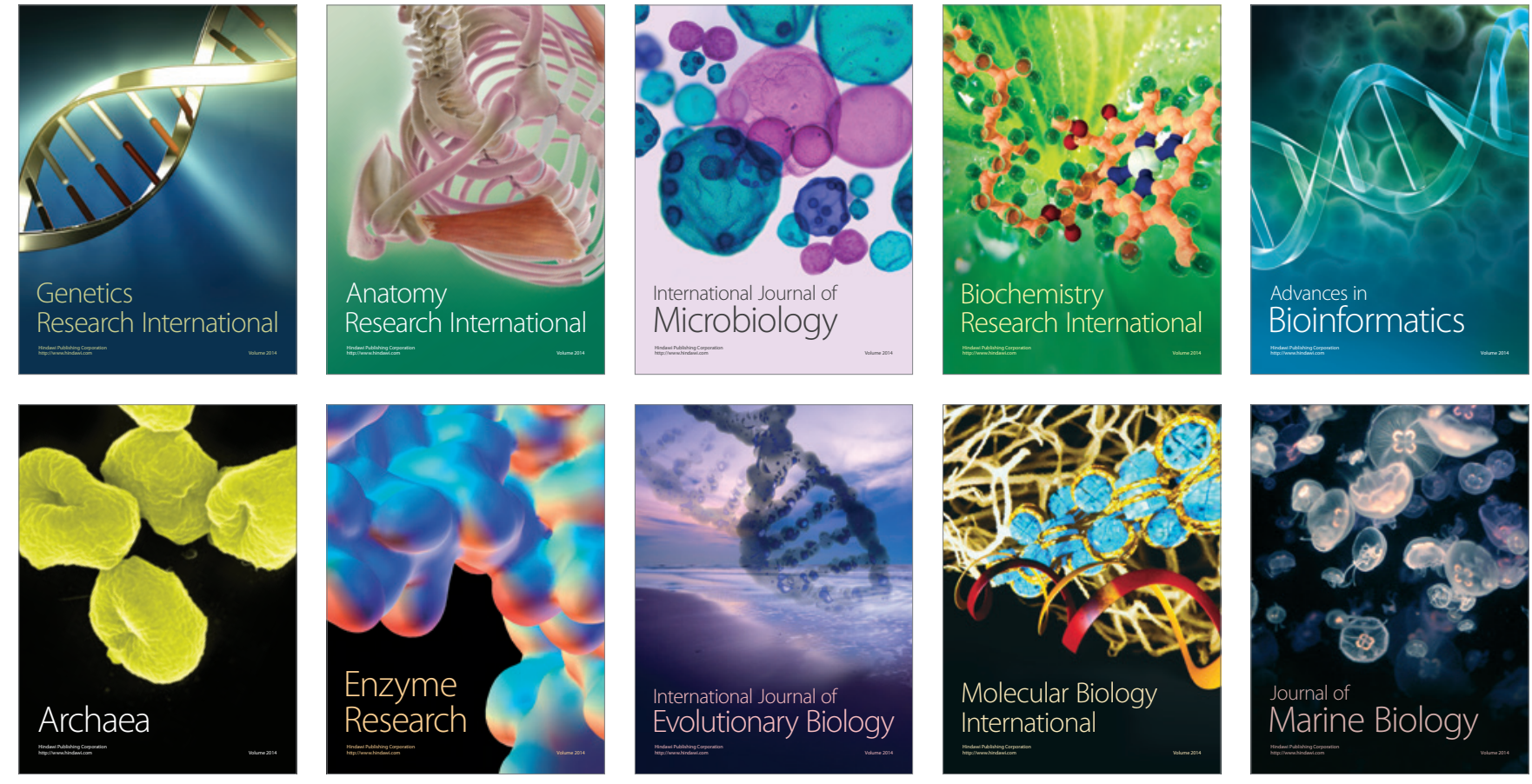